\title{
Prenatal and Postnatal Diagnostics of a Child with Bardet-Biedl Syndrome: Case Study
}

\author{
Magdalena Pasińska ${ }^{1-3 *}$, Lech Dudarewicz ${ }^{4}$, Lucjusz Jakubowski ${ }^{4}$ and Olga Haus ${ }^{5}$ \\ ${ }^{1}$ Department of Clinical Genetics, Faculty of Medicine, Collegium Medicum in Bydgoszcz, Nicolaus Copernicus University, Poland \\ ${ }^{2}$ Genetic Counseling, University of Medicine, Poland \\ ${ }^{3}$ Department of Obstetrics, Gynecology and Oncology, Faculty of Medicine, Collegium Medicum, Nicolaus Copernicus University, Poland \\ ${ }^{4}$ Department of Genetics, Polish Mother's Memorial Hospital, Poland \\ ${ }^{5}$ Department of Hematology, Blood Malignancies and Bone Marrow Transplantation, University of Medicine, Poland
}

*Corresponding author:Magdalena Pasińska, Department of Genetics, Collegium Medicum in Bydgoszcz Skłodowskiej - Curie 9, 85-094 Bydgoszcz, Poland, Tel: +48 5258536 70; Fax: + 485258535 68; E-mail: magdalena@cm.umk.pl, magdapas@wp.pl

Received date: October 02, 2015; Accepted date: November 24, 2015; Published date: December 01, 2015

Copyright: ( 2015 Pasińska M, et al. This is an open-access article distributed under the terms of the Creative Commons Attribution License, which permits unrestricted use, distribution, and reproduction in any medium, provided the original author and source are credited.

\begin{abstract}
Bardet Biedl Syndrome (BBS) is a rare, genetically determined syndrome which can result from a mutation in one of 19 known genes (often called BBS complex), which play a vital role in building structures and cell functions of cilia. Phenotypic symptoms of the syndrome usually progress in the first decade of life, however, they are characterized by a high diversity which makes their diagnosis difficult, especially in the early stage of life. The diagnosis is most frequently based on clinical symptoms and established in late childhood or adult life. BBS is a disorder of the non-motile cilia, which play the role of antennae receiving and transmitting sensory signals to photoreceptors of the retina, hearing cells or olfactory cells. Clinical symptoms of the disorders affecting these structures are: retinal pigmentary degeneration, polydactyly, learning difficulties, and formation of kidney, liver and pancreas cysts. The inheritance mode of BBS is in $80 \%$ autosomal recessive, which is connected with both parents carrying a mutated allele, and usually only giving birth to a symptomatic child defines a family as being at genetic risk, whith $25 \%$ risk of having another child with the disease and $50 \%$ of having offspring that are asymptomatic carriers For families with an identified mutation there exists a possibility of conducting prenatal or preimplementation genetic diagnosis in subsequent pregnancies The article presents a case of a patient in whom prenatal ultrasonography and subsequent clinical trials in post-natal period resulted in a Bardet-Biedl syndrome diagnosis, which was later confirmed through molecular tests.
\end{abstract}

Keywords: Prenatal diagnosis; Ultrasound; Genetic syndromes; Bardet-Biedl syndrome

\section{Introduction}

Bardet-Biedl syndrome (BBS) is a rare, genetically determined syndrome. It can result from a mutation in one of the 19 known genes (often called BBS complex), which play a vital role in building structures and cell functions of cilia [1,2]. Cilia are specialized hairshaped structures which are located on the surface of most nonproliferating eukaryotic cells. Motile cilia are used for movement of fluids, such as the removal of mucus and pollution from lung epithelial cells. Motile cilia diseases may result in bronchiectasia, infertility or left-right asymmetry of the body [3]. BBS is a disorder of the nonmotile cilia. Non-motile cilia function as antennae that receive and transmit sensory signals to photoreceptors of the retina, cells of hearing or olfactory cells. They play a critical role during development and in maintaining cellular and tissue homeostasis. For mammals primary cilia appear to be even more important to the development, morphogenesis, and signalling in the brain than for any other organisms. Clinical symptoms of the disorders affecting these structures involve: retinal pigmentary degeneration, polydactyly, learning difficulties, and formation of kidney, liver and pancreas cysts [4].
BBS occurrence varies among different populations, from 1:160 000 in northern Europe to 1:13500 in Kuwait. The increased occurrence in Kuwait is caused by a high number of marriages between relatives. Phenotypic symptoms of the syndrome usually progress in the first decade of life, however, as they are characterized by high diversity, the diagnosis proves to be difficult - especially in the early stages of life. The diagnosis is most frequently based on clinical symptoms and established in late childhood or adult life [5]. The inheritance mode of BBS is in $80 \%$ autosomal recessive, which is related to both parents carrying a mutated allele. In most of the cases, only giving birth to a symptomatic child defines a family as being at genetic risk, with $25 \%$ chance of having another child with the disease and $50 \%$ of having offspring that are asymptomatic carriers [5,6]. For families with an identified mutation there exists a possibility of conducting prenatal or pre-implantation genetic diagnosis in subsequent pregnancies [5-7]. In $10 \%$ of cases the inheritance is triallelic, in which three mutations from genes at two different BBS loci segregate with the expression of the disease. In this type of inheritance the confirmation through molecular diagnosis is either difficult or impossible [7,8].

The article presents a case of a patient for whom prenatal ultrasonography and subsequent clinical evaluation in the postnatal period resulted in the diagnosis of Bardet-Biedl syndrome. This was later confirmed by molecular tests. 
Citation: Pasinska M, Dudarewicz L, Jakubowski L, Haus O (2015) Prenatal and Postnatal Diagnostics of a Child with Bardet-Biedl Syndrome: Case Study. J Mol Genet Med 9: 189. doi:10.4172/1747-0862.1000189

Page 2 of 5

\section{Case Description}

\section{Prenatal period}

A 27-year-old woman was referred to a genetic clinic for consultation in the 15 th week of her 2 nd pregnancy. The reason for being referred was bilateral pyelectasis, hyperechogenic bowel and nuchal edema of the fetus. Medical history of both the patient and her husband was not burdened with any congenital disorders nor intellectual disabilities, neither were they relatives. Her first pregnancy ended in the 40th week with a natural birth of a healthy female child. Due to ultrasound abnormalities, fetal karyotyping by amniocentesis was performed, showing the standard 46, XX female karyotype. Biochemical testing of the amniotic fluid for presence of acetylcholinesterase (ACHE) allowed excluding an open neural tube defect. Ultrasound scan conducted in the 19th week of the pregnancy showed bilateral nephromegaly with a hyperechogenic cortical layer of the kidneys as well as a slightly dilated pelvicalyceal system.

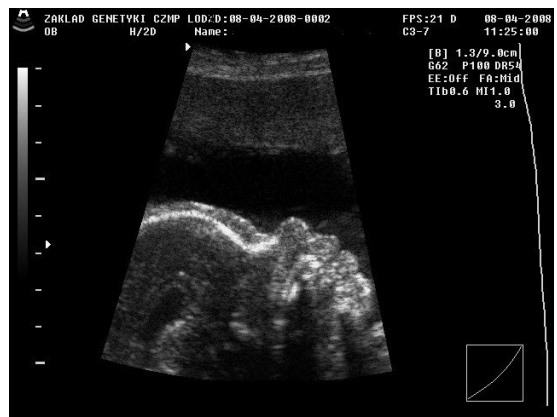

Figure 1: Abnormal facial profile-low nasal bridge in the fetus with Bardet-Biedl syndrome in the 26th week of pregnancy.

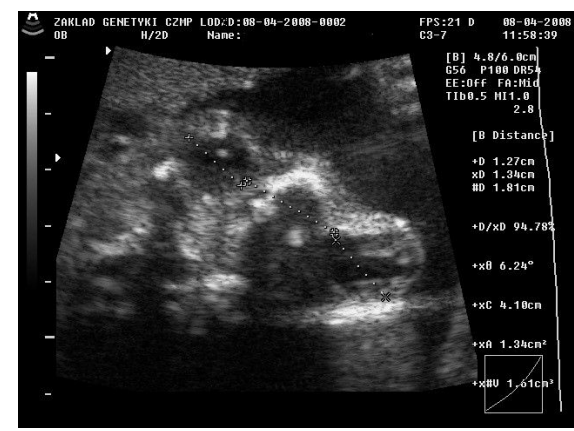

Figure 2: Hypertelorism in the fetus with Bardet-Biedl syndrome in the 26 th week of pregnancy.

During the 26th week of the pregnancy, ultrasonography showed slight dolichocephaly, an abnormal facial profile - low nasal bridge (Figure 1), hypertelorism (Figure 2). There was a noticeable nuchal edema, with the nuchal fold equalling $11 \mathrm{~mm}$. The feet were described as difficult to assess. There was a mild disproportion of the heart in favour of the right side, small amount of fluid was visible in the pericardium. The study revealed massive ascites, with slightly hyperechogenic bowels, without evident dilatation. Mild pyelectasia and significant nephromegaly was also evident (Figure 3 ).

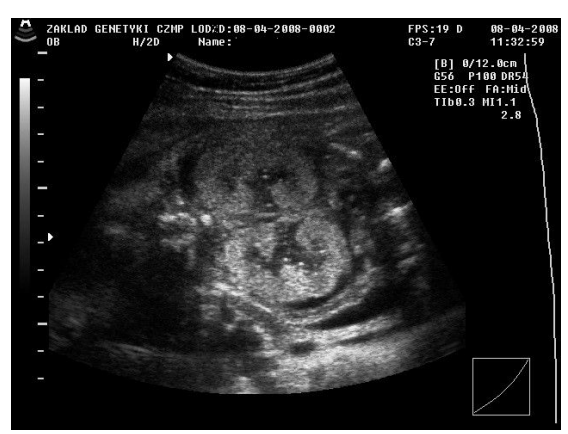

Figure 3: Transverse scan showing pelvicalyceal systems of the fetus slightly englarged, a significant nephromegaly of both kidneys of the fetus with the Bardet - Biedl syndrome in 26th week of pregnancy.

\section{Postnatal period}

In the 35th week of the pregnancy the infant was born in a generally poor condition, with Apgar score of 1 . Her birth weight was $3380 \mathrm{~g}$ (>90c) and she was delivered through caesarean section due to deteriorating cardiotocographic (CTG) tracing and with accumulating ascites. The pregnancy was also complicated with diabetes mellitus. After birth the infant was diagnosed with pectus excavatum with the narrowing of the thorax in proportion to the abdomen, polycystic left kidney, bilateral dilation of the pelvicalyceal system, cystoid areas behind the urethra, facial dysmorphism, polydactyly of the feet, a vestigial postaxial polydactyly in the right hand, and tricuspid valve insufficiency. The neonatal period was complicated with respiratory insufficiency (the child was intubated on the first day of life and stayed on mechanical ventilation for nine days) and a generalized infection with staphylococci. Due to the elevation of the diaphragm resulting in dyspnea, the decompression of two cysts was performed. The child was then transferred to a paediatric surgery clinic for further diagnostics and treatment.

During the subsequent diagnosis a $2^{\text {nd }}$ grade virilisation of genital organs was observed: clitoromegaly, an urogenital sinus, and hydrocolpos. For this reason urine steroid profile was analysed, which excluded blocks of adrenal steroidogenesis.

Hydrocolpos was decompressed twice using cystourethrovaginoscopy, and on the 24th day of life a reconstruction of the urogenital sinus was performed. During hospitalization a renal insufficiency was detected, along with ionic disorders and a tachycardia of atrial origin.

\section{Early childhood}

A genetic syndrome, either McKusick-Kaufman or Bardet-Biedl was suspected on the basis of the clinical symptoms. Therefore, a molecular diagnosis of BBS10 gene mutation was indicated. The diagnosis was performed in Genesis Medical Diagnostic Centre in Poznań. Based on the performed analysis, heterozygotic mutations, c.C145(pR49W), in exon 1 and c.T589C(p.Y197H) mutation in exon 2 of BBS10 were discovered, and this confirmed the clinical diagnosis of BBS.

In her 26th month of life the girl weighed $19 \mathrm{~kg}(>97 \mathrm{c})$ with the height of $89 \mathrm{~cm}(<3 \mathrm{c})$. She started walking at the age of two, while her 
first words came at the age of three. The physical examination revealed megacephaly, a round face with a low nasal bridge, an epicanthal fold, hypertelorism, low set ears, numerous melanodermata, obesity and an overdeveloped adipose tissue.

The girl slept during the day and had problems with sleeping at night. She had painful crural cramps, initially happening every day, and then recurring once a month. The patient experienced tonic muscle tension of the whole body without convulsion followed by crying. Neurological examination revealed neurodevelopmental retardation and physical sluggishness, without distinct central nervous system focal injuries. On the basis of electroencephalography, disorders with epileptic etiology were ruled out. Ophthalmological tests showed bilateral hyperopic astigmatism.

A pedagogical and psychological testing conducted after the third birthday disclosed a lowered motor development in comparison to the age norm. The girl sat, walked self-dependently but more heavily than her peers, didn't try to jump and couldn't stand on one leg. She was not responding to commands, nor did she try to imitate syllables or simple words. She didn't attempt to identify objects by their names. According to age norms, the girl displayed a lowered social and emotional contact. She didn't cooperate, forced her own activity, tended to be stubborn and moody, and got offended easily, cried and evaded human contact. She was diagnosed with a lowered accuracy of visual and auditory perception. A speech examination identified congenital developmental dysphasia caused by endogenic factors. The girl had a perceptive-expressive disorder with a speech development delay.

At the age of four the child's weight was $24 \mathrm{~kg}(>97 \mathrm{c})$ and the height $90 \mathrm{~cm}(<3 \mathrm{c})$, and at the age of 4 and a half the girl weighed $28 \mathrm{~kg}$ $(>97 \mathrm{c})$ and measured $100 \mathrm{~cm}(<3 \mathrm{c})$ (Figures 4 and 5).

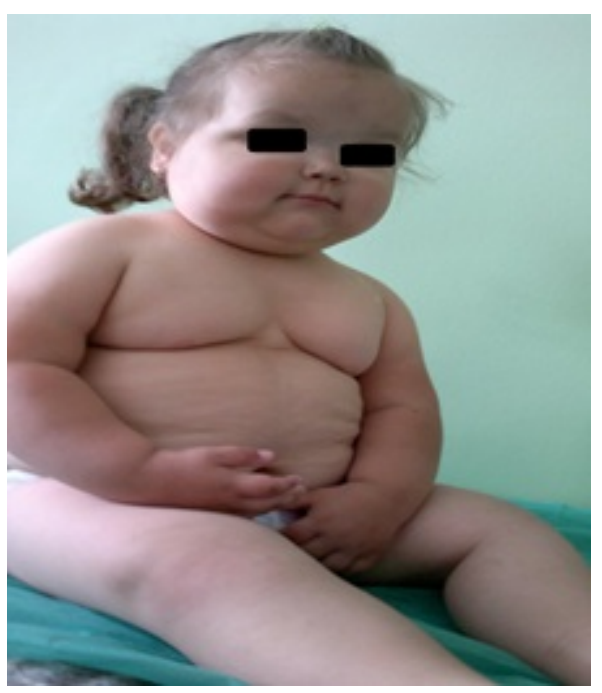

Figure 4: Obesity and mecrcephaly in a girl with Bardet - Biedl syndrome in fourth years of life.

Since her birth, the child had frequent occurrences of bronchitis and pneumonia, as well as persistent auscultatory changes such as wheezing and buzz. Based on a bronchoscopic material ciliary dyskinesia was diagnosed. Additionally, the patient was also observed to have a midline epiglottis split, which could be the reason for the frequent cases of bronchitis, as food was entering the airways during food intake.

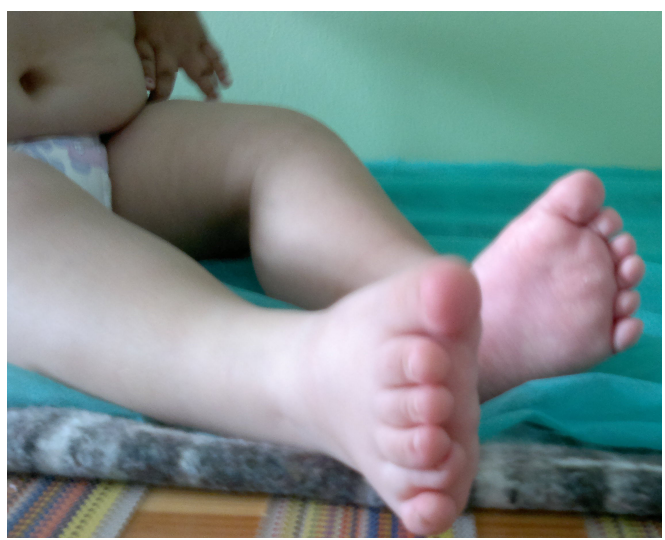

Figure 5: Polydactyly of both feet in the girl with Bardet-Biedl syndrome in the fourth year of life.

Then the chronic renal insufficiency reached the third stage of severity. Bilateral hydronephrosis developed, with concommitant hyperchloremic metabolic acidosis, reduced glomerular filtration rate, impaired renal tubes, mixed hyperlipidemia with a slightly elevated urea nitrogen levels, elevated liver enzymes and anemia. At present the girl doesn't signal physiological needs.

\section{Discussion}

Molecular testing of the BBS10 gene conducted at the age of 2,5 years revealed a complex heterozygous mutation c.C145T (p.R49W) in exon, and c.T589C (p.Y197H) mutation in exon 2. This confirmed the clinical diagnosis of Bardet-Biedl syndrome. A rapid development of molecular testing in the last decade has led to the discovery of changes in the 19 genes in patients with clinically diagnosed BBS, encompassing missense, nonsense and frameshift mutations [6]. The BBS10 gene is located on the long arm of the chromosome 12(12q21.2) $[7,9]$.

The BBS10 gene provides instructions for making a protein that is found in many types of cells. The BBS10 protein is part of a group (complex) of proteins that functions as a chaperonin. It helps other proteins fold into their correct 3-dimensional shapes so they can perform their usual functions. The BBS10 protein also helps fold or stabilize certain proteins that are necessary for the normal formation of cilia $[9,10]$.

Certain regional differences in the frequency of pathogenic mutations occur in Northern Europe. The most frequent changes occur in the BBS1 and BBS10 genes which constitute $2,2 \%$ and $20 \%$ of cases respectively, while in patients from East, Central and North Africa the most frequent changes are situated in BBS, BBS4 and BBS6 genes $[2,7,9]$.

Due to the presence of hydrometrocolpos and vaginal atresia concurrently with the classical BBS phenotype, molecular diagnostic procedures were initiated with the analysis of the BBS10 gene.

In the study by Billinsky et al. conducted in 93 patients of various ethnic origin, it was observed that the presence of mutations of the chaperonin-like BBS genes (BBS6, BBS10 and BBS12), apart from the 
classical BBS phenotype, induces the traits of MKKS, including congenital heart defects, vaginal atresia, hydrometrocolpos and cryptorchidism, as well as the traits of Alström syndrome, including diabetes, loss of hearing, hepatic and endocrine disorders, and cardiomyopathy [10].

Forsythe et al., in studies comparing cardiovascular risk factors, showed a connection between the phenotype and the type of BBS1 and BBS10 mutations. These studies demonstrated that in patients with the BBS1 gene mutations the risk of cardiovascular disease is lower than in patients with mutations of the BBS10 gene [2].

In the presented case, bilaterally enlarged kidneys without corticomedullary differentiation were revealed during the prenatal ultranosound scan which took place as early as in her 15th week of prenatal life. Later, renal cysts were discovered as well as a mild dilatation of the pelvicalyceal system and increasing ascites [11]. Based on the ultrasound image, a genetically conditioned disorder was suspected, i.e. Perlman and Simpson - Golabi - Behmel syndromes were considered in the differential diagnosis. Since hexadactyly was not detected prenatally, an autosomal recessive polycystic kidney disease (ARPKD) was also taken into consideration. BBS-related pathological changes in kidneys are observed in the prenatal period and affect $30-100 \%$ of patients $[1,12]$. Lesions in other organs causing further symptoms, such as progressive chromatic retinopathy, obesity, developmental delay, hepatic fibrosis and dental changes were observed later. Hyperechogenic kidneys can be diagnosed as early as late first trimester. In the second trimester the kidneys are considered abnormal, when they are more echogenic than the liver or spleen [13]. Abnormal echogenicity of the kidneys is associated with numerous microscopic cysts, dysplasia and tubular dilatation. Cystic renal disease should be suspected in the case of large cysts but also in the case of hyperechogenic kidneys. Therefore, the differential diagnosis should include a family history check with a focus on the syndrome occurrence as well as coexistent defects [11-13].

According to observations made by Cassart et al. the size of kidneys and corticomedullary differentiation in the BBS normalizes before the second year of life. Other authors also observed a regression of renal cysts [13]. However, renal insufficiency occurs in 5-25\% of BBS cases, and in $4-10 \%$ of cases the insufficiency is progressive, which is the most frequent cause of death $[14,15]$.

McKusick-Kaufman syndrome, which is characterized by a triade of symptoms: postaxial polydactyly, congenital heart disease and genital defects (most often observed in boys) was also proposed in differential diagnosis. Diagnosis of this syndrome is based mostly on clinical criteria and, due to a better prognosis, requires a priori exclusion of BBS $[6,15]$. One of the known genes responsible for this syndrome is the MKS gene. Because of additional clinical symptoms appearing with age in the McKusic-Kaufman syndrome, a diagnosis should not be given before the 5th year of life unless there is a possibility of a molecular confirmation of the syndrome $[6,15]$.

In an ultrasound scan an anechoic mass filling the pelvis behind the urinary bladder of the fetus was observed. After birth hydrometrocolpos was diagnosed. In fetuses it appears as a large cyst in the abdomen reaching the lower pelvis. It is caused by the distension of the uterine cavity and the vagina due to an accumulation of cervical gland secretions stimulated by maternal and placental estrogen in the presence of the vaginal septation or atresia or an imperforate hymen $[6,16]$.
Although BBS is associated with immotile ciliary syndrome, ciliary dyskinesia syndrome was diagnosed and confirmed in the patient by the analysis of tissue sections and assessment of cilia using transmission electron microscopy (TEM). A properly built cilium is composed of the system of microtubules: nine pairs located peripherally and one pair located centrally. Every peripheral pair of microtubules is accompanied by dynein, a protein forming the socalled outer and inner arms. Normally, dynein allows cilia to perform coordinated movement. Pathological symptoms are caused by the abnormal structure of the cilia present on the human epithelia. The disorder is usually inherited in an autosomal recessive manner $[3,4]$. In the presented patient, ciliary dyskinesia might have been secondary and caused by a recurring inflammation of the respiratory tract. However, a coexisting disorder with similar inheritance cannot be excluded.

Although the ocular changes are impossible to detect in early childhood, a progressing retinal dystrophy is one of the most frequently occurring symptoms in BBS $(69-100 \%)[16,17]$. Most often it is a rod-cone dystrophy, where central vision is impaired at first, followed by peripheral vision and night vision impairment. A decline of chromatic vision occurs early. Measuring the BBS-patients' field of vision is difficult due to mental retardation, age, and frequent lack of cooperation. Electroretinography is a procedure which allows an early retinopathy recognition when changes in the retina are minimal and still barely perceptible $[1,17]$. Even though there is no causal treatment for BBS, an early diagnosis is important for the necessity of designing a diet, monitoring of glycaemic levels, arterial blood pressure and kidney and liver parameters as well as ophthalmological examination and psychological help to alleviate the symptoms and improve child's development [2]. The increased frequency of obesity, hypertension, diabetes and kidney cancer has been found in heterozygotic carriers of the BBS gene mutation [2,7]. Therefore, prophylactic procedures are necessary for relatives of patients with this syndrome $[2,16]$.

During pregnancy ultrasound examination can identify many genetic syndromes characterized by similar clinical symptoms. These disorders are often inherited in an autosomal recessive manner. Some of them, however, may be lethal, like in the case of Meckel-Gruber syndrome [17]. Therefore, BBS should also be included in the differential diagnostics of the syndromes characterized by the presence of echogenic kidneys, without familial history. This information should be considered in further decisions regarding invasive prenatal diagnostics and other diagnostic methods. Diagnosis of a pathogenic mutation in the patient allows the identification of healthy relatives as a carrier, which later helps when seeking genetic advice and facilitates planning further procreation. Identification of the mutation allows earlier employment of invasive prenatal molecular diagnostics of chorionic villi (CVS), or even preimplantation diagnosis (PGD). However, if no mutation is identified in a deceased child or fetus with findings suggestive of this disorder, it is advisable to store his/her genetic material that may allow identification of the pathogenic lesion and proper diagnosis $[1,9]$.

\section{References}

1. Forsythe E, Beales PL (2013) Bardet-Biedl syndrome. Eur J Hum Genet 21: 8-13.

2. Forsythe E, Sparks K, Hoskins BE, Bagkeris E, McGowan BM, et al. (2015) Genetic predictors of cardiovascular morbidity in Bardet-Biedl syndrome. Clin Genet 87: 343-349. 
Citation: Pasinska M, Dudarewicz L, Jakubowski L, Haus O (2015) Prenatal and Postnatal Diagnostics of a Child with Bardet-Biedl Syndrome: Case Study. J Mol Genet Med 9: 189. doi:10.4172/1747-0862.1000189

Page 5 of 5

3. Loktev AV, Jackson PK (2013) Neuropeptide Y family receptors traffic via the Bardet-Biedl syndrome pathway to signal in neuronal primary cilia. Cell Rep 5: 1316-1329.

4. Novas R, Cardenas-Rodriguez M, Irigoín F, Badano JL (2015) BardetBiedl syndrome: Is it only cilia dysfunction? FEBS Lett 589: 3479-3491.

5. Dar P, Sachs GS, Carter SM, Ferreira JC, Nitowsky HM, et al. (2001) Prenatal diagnosis of Bardet-Biedl syndrome by targeted secondtrimester sonography. Ultrasound Obstet Gynecol 17: 354-356.

6. Gaucherand P, Vavasseur-Monot C, Ollagnon E, Boisson C, Labaune JM, et al. (2002) McKusik-Kaufman syndrome: prenatal diagnosis, genetics and follow up. Prenat Diagn 22: 1048-1050.

7. Sapp JC, Nishimura D, Johnston JJ, Stone EM, Héon E, et al. (2010) Recurrence risks for Bardet-Biedl syndrome: Implications of locus heterogeneity. Genet Med 12: 623-627.

8. Karmous-Benailly H, Martinovic J, Gubler MC, Sirot Y, Clech L, et al (2005) Antenatal presentation of Bardet-Biedl syndrome may mimic Meckel syndrome. Am J Hum Genet 76: 493-504.

9. M'hamdi O, Ouertani I, Chaabouni-Bouhamed H (2014) Update on the genetics of bardet-biedl syndrome. Mol Syndromol 5: 51-56.

10. Sinha S, Belcastro M, Datta P, Seo S, Sokolov M (2014) Mutations in chaperonin-like BBS genes are a major contributor to disease development in a multiethnic Bardet-Biedl syndrome patient population. J Med Genet 47: 453-463.

11. Bergmann C (2015) ARPKD and early manifestations of ADPKD: the original polycystic kidney disease and phenocopies. Pediatr Nephrol 30: 15-30.

12. Kute VB, Vanikar AV, Gumber MR, Patel HV, Shah PR, et al. (2013) Bardet-biedl syndrome: a rare cause of chronic kidney disease. Indian J Clin Biochem 28: 201-205.

13. Cassart M, Eurin D, Didier F, Guibaud L, Avni EF (2004) Antenatal renal sonographic anomalies and postnatal follow-up of renal involvement in Bardet-Biedl syndrome. Ultrasound Obstet Gynecol 24: 51-54.

14. Chaumoitre K, Braun M, Cassart M, Maugey-Laulom B, Euris D et al. (2006). Differential diagnosis of fetal hypoechogenic cystic kidneys unrelated to renal tract anomalies: a multicenter study. Ultrasouns Obstet Gynecol 28: 911-917.

15. Rodriguez MM (2014) Congenital Anomalies of the Kidney and the Urinary Tract (CAKUT). Fetal Pediatr Pathol 33: 293-320.

16. Slavotinek A, Beales P (2011) Clinical utility gene card for: Bardet-Biedl syndrome. Eur J Hum Genet 19.

17. Santos PR, Monteiro DL, de Paula PH, Monte Neto VL (2014) Report of four cases of Bardet-Biedl syndrome. J Bras Nefrol 36: 250-253. 